\title{
A GKM description of the equivariant cohomology ring of a homogeneous space
}

\author{
V. Guillemin · T. Holm • C. Zara
}

Received: April 23, 2004 / Revised: April 28, 2005 / Accepted: May 19, 2005

(C) Springer Science + Business Media, Inc. 2006

\begin{abstract}
Let $T$ be a torus of dimension $n>1$ and $M$ a compact $T$-manifold. $M$ is a GKM manifold if the set of zero dimensional orbits in the orbit space $M / T$ is zero dimensional and the set of one dimensional orbits in $M / T$ is one dimensional. For such a manifold these sets of orbits have the structure of a labelled graph and it is known that a lot of topological information about $M$ is encoded in this graph.

In this paper we prove that every compact homogeneous space $M$ of non-zero Euler characteristic is of GKM type and show that the graph associated with $M$ encodes geometric information about $M$ as well as topological information. For example, from this graph one can detect whether $M$ admits an invariant complex structure or an invariant almost complex structure.
\end{abstract}

Keywords GKM graph · Homogeneous spaces · Equivariant cohomology

\section{Introduction}

Let $T$ be a torus of dimension $n>1, M$ a compact manifold,

$$
\tau: T \times M \rightarrow M
$$

a faithful action of $T$ on $M$, and $M / T$ the orbit space of $\tau . M$ is called a GKM manifold if the set of zero dimensional orbits in the orbit space $M / T$ is zero dimensional and the set

\footnotetext{
V. Guillemin

Department of Mathematics, MIT, Cambridge, MA 02139

e-mail:vwg@math.mit.edu

\section{T. Holm}

Department of Mathematics, University of Connecticut, Storrs, CT 06268

e-mail: tsh@math.uconn.edu

C. Zara $(\square)$

Department of Mathematics, Penn State Altoona, PA, 16601

e-mail: czara@psu.edu
} 
of one dimensional orbits in $M / T$ is one dimensional. Under these hypotheses, the union, $\Gamma \subset M / T$, of the set of zero and one dimensional orbits has the structure of a graph: Each connected component of the set of one-dimensional orbits has at most two zero-dimensional orbits in its closure; so these components can be taken to be the edges of a graph and the zero-dimensional orbits to be the vertices. Moreover, each edge, $e$, of $\Gamma$ consists of orbits of the same orbitype: namely, orbits of the form $\mathcal{O}_{e}=T / H_{e}$, where $H_{e}$ is a codimension one subgroup of $T$. Hence one has a labelling

$$
e \rightarrow H_{e}
$$

of the edges of $\Gamma$ by codimension one subgroups of $T$.

It has recently been discovered that if $M$ has either a $T$-invariant complex structure or a $T$-invariant symplectic structure, the data above - the graph $\Gamma$ and the labelling [1.1]contain a surprisingly large amount of information about the global topology of $M$. For instance, Goresky, Kottwitz, and MacPherson proved that the ring structure of the equivariant cohomology ring $H_{T}^{*}(M)=H_{T}^{*}(M ; \mathbb{C})$ is completely determined by this data, and Knutson and Rosu have shown that the same is true for the $\operatorname{ring} K_{T}(M) \otimes \mathbb{C}$.

The manifolds $M$ which we will be considering below will be neither complex nor symplectic; however we will make an assumption about them which is in some sense much stronger then either of these assumptions. We will assume that $T$ is the Cartan subgroup of a compact, semisimple, connected Lie group $G$, and that $G$ acts transitively on $M$, i.e. $M$ is a $G$-homogeneous space. There is a simple criterion for such a manifold to be a GKM manifold.

Theorem 1.1. Suppose $M$ is a G-homogeneous manifold. Then the following are equivalent.

(1) The action of $T$ on $M$ is a GKM action;

(2) The Euler characteristic of $M$ is non-zero;

(3) $M$ is of the form $M=G / K$, where $K$ is a closed subgroup of $G$ containing $T$.

If $K$ is connected, then $K$ is the identity component of the centralizer in $G$ of the center of $K$, which in this case is the same as the identity component of the normalizer in $G$ of the center of $K$ (see [2]).

As we mentioned above, the data [1.1] determine the ring structure of $H_{T}^{*}(M)$ if $M$ is either complex or symplectic. This result is, in fact, true modulo an assumption which is weaker than either of these assumptions; and this assumption - equivariant formality-is satisfied by homogeneous spaces which satisfy the hypotheses of the theorem. Hence, for these spaces, one has two completely different descriptions of the ring $H_{T}^{*}(M)$ : the graph theoretical description above and the classical Borel description. In Section 2 we will compute the graph $\Gamma$ of a space $M$ of the form $G / K$, with $T \subset K$, and show that it is a homogeneous graph, i.e. we will show that the Weyl group of $G, W_{G}$, acts transitively on the vertices of $\Gamma$ and that this action preserves the labelling [1.1]. We will then use this result to compare the two descriptions of $H_{T}^{*}(M)$.

One of the main goals in this paper is to show that for homogeneous manifolds $M$ of GKM type, some important features of the geometry of $M$ can be discerned from the graph $\Gamma$ and the labelling (1.1). One such feature is the existence of a $G$-invariant almost complex structure. The subgroups, $H_{e}$, labelling the edges of $\Gamma$ are of codimension one in $T$; so, up to sign, they correspond to weights, $\alpha_{e}$, of the group $T$. It is known that the $W_{K}$-invariant 
labelling (1.1) can be lifted to a $W_{K}$-invariant labelling

$$
e \rightarrow \alpha_{e}
$$

if $M$ is a coadjoint orbit of $G$ (hence, in particular, a complex $G$-manifold). Moreover, this labelling has certain simple properties which we axiomatize by calling a map with these properties an axial function (see Section 3.1). In Section 3 we prove the following result.

Theorem 1.2. The homogeneous space $M$ admits a $G$-invariant almost complex structure if and only if $\Gamma$ possesses a $W_{K}$-invariant axial function (1.2) compatible with (1.1).

This raises the issue: Is it possible to detect from the graph theoretic properties of the axial function (1.2) whether or not $M$ admits a $G$-invariant complex structure? Fix a vector $\xi \in \mathfrak{t}$ such that $\alpha_{e}(\xi) \neq 0$ for all oriented edges, $e$, of $\Gamma$, and orient these edges by requiring that $\alpha_{e}(\xi)>0$. We prove in Section 4 the following theorem.

Theorem 1.3. A necessary and sufficient condition for $M$ to admit a $G$-invariant complex structure is that there exist no oriented cycles in $\Gamma$.

\section{Remarks:}

(1) $M$ admits a $G$-invariant complex structure if and only if it admits a $G$-invariant symplectic structure; and, by the Konstant-Kirillov theorem, it has either (and hence both) of these properties if and only if it is a coadjoint orbit of $G$.

(2) By the Goresky-Kottwitz-MacPherson theorem, the graph $\Gamma$ and the labelling (1.1) determine the cohomology ring structure of $M$. The additive cohomology of $M$, i.e. its Betti numbers, $\beta_{i}$, can be computed from (1.2) by the following simple recipe: For each vertex, $p$, of the graph $\Gamma$, let $\sigma_{p}$ be the number of oriented edges issuing from $p$ with the property that $\alpha_{e}(\xi)<0$. Then

$$
\beta_{i}= \begin{cases}0, & \text { if } i \text { is odd, } \\ \#\left\{p ; \sigma_{p}=i / 2\right\}, & \text { if } i \text { is even. }\end{cases}
$$

(3) To streamline the exposition of the material above, we will assume from now on that $K$ is connected, or, equivalently, that $G / K$ is simply connected. However, many of the results of this paper (for instance Theorems 1.2 and 1.3) are true without this assumption and can be deduced from the results in the simply connected case by covering space arguments.

One question we have not addressed in this paper is the question: When is a labelled graph the GKM graph of a homogeneous space of the form $G / K$ with $T \subset K$ ? For some partial answers to this question see [11]. 


\section{The equivariant cohomology of homogeneous spaces}

\subsection{The Borel description}

Let $G$ be a compact semi-simple Lie group, $T$ a Cartan subgroup of $G, K$ a connected, closed subgroup of $G$ such that

$$
T \subset K \subset G
$$

and let $\mathfrak{t} \subset \mathfrak{k} \subset \mathfrak{g}$ be the Lie algebras of $T, K$, and $G$.

Let $\Delta_{K} \subset \Delta_{G}$ be the roots of $K$ and $G$, with $\Delta_{K}^{+} \subset \Delta_{G}^{+}$sets of positive roots, let

$$
\Delta_{G, K}=\Delta_{G}-\Delta_{K},
$$

and let $W_{K} \subset W_{G}$ be the Weyl groups of $K$ and $G$. We will regard an element of $W_{G}$ both as an element of $N(T) / T$ and as a transformation of the dual Lie algebra $t^{*}$ (or as a transformation of $\mathfrak{t}$, via the isomorphism $\mathfrak{t}^{*} \simeq \mathfrak{t}$ given by the Killing form). Also, we will assume for simplicity that $G$ is simply connected and that the homogeneous space $G / K$ is oriented.

Now suppose $M$ is a $G$-manifold. Then the equivariant cohomology $\operatorname{ring} H_{T}^{*}(M)$ is related to the cohomology ring $H_{G}^{*}(M)$ by

$$
H_{T}^{*}(M)=H_{G}^{*}(M) \otimes_{\mathbb{S}\left(\mathfrak{t}^{*}\right)^{W_{G}}} \mathbb{S}\left(\mathfrak{t}^{*}\right)
$$

(see [8], Chap. 6), where $\mathbb{S}\left(\mathrm{t}^{*}\right)$ is the symmetric algebra of $\mathrm{t}^{*}$. In particular, let $M=G / K$, where $K$ acts on $G$ by right multiplication. Then $G$ acts on $M$ by left multiplication and

$$
H_{G}^{*}(M)=H_{G}^{*}(G / K)=\mathbb{S}\left(\mathfrak{k}^{*}\right)^{K}=\mathbb{S}\left(\mathfrak{t}^{*}\right)^{W_{K}},
$$

hence

$$
H_{T}^{*}(G / K)=\mathbb{S}\left(\mathfrak{t}^{*}\right)^{W_{K}} \otimes_{\mathbb{S}\left(\mathfrak{t}^{*}\right)^{W_{G}}} \mathbb{S}\left(\mathfrak{t}^{*}\right)
$$

This is the Borel description of $H_{T}^{*}(G / K)$. Throughout this paper, unless stated otherwise, $M$ is the homogeneous space $G / K$.

\subsection{The GKM graph of $M$}

In the following subsections, we will show that $M$ is equivariantly formal and is a GKM space. Then we will relate the the GKM description of the equivariant cohomology ring of $M$ to the description above.

\subsubsection{Equivariant formality}

The $\mathbb{S}\left(\mathrm{t}^{*}\right)$-module structure of the equivariant cohomology ring $H_{T}^{*}(M)$ can be computed by a spectral sequence (see [8], p. 70) whose $E_{2}$ term is $H(M) \otimes \mathbb{S}\left(t^{*}\right)$, and if this spectral 包 Springer 
sequence collapses at this stage, then $M$ is said to be equivariantly formal. If $M=G / K$, with $T \subset K$, then,

$$
H^{\text {odd }}(M)=0,
$$

(see [5], p. 467), and from this it is easy to see that all the higher order coboundary operators in this spectral sequence have to vanish by simple degree considerations. Hence $M$ is equivariantly formal. One implication of equivariant formality is the following:

Theorem 2.1. The restriction map

$$
H_{T}^{*}(M) \rightarrow H_{T}^{*}\left(M^{T}\right)
$$

is injective.

Proof: By a localization theorem of Borel (see [1]), the kernel of (2.2) is the torsion submodule of $H_{T}^{*}(M)$. However, if $M$ is equivariantly formal, then $H_{T}^{*}(M)$ is free as an $\mathbb{S}\left(t^{*}\right)$-module, so the kernel has to be zero.

Thus $H_{T}^{*}(M)$ imbeds as a subring of the ring

$$
H_{T}^{*}\left(M^{T}\right)=H^{0}\left(M^{T}\right) \otimes_{\mathbb{C}} \mathbb{S}\left(\mathfrak{t}^{*}\right)
$$

We will give an explicit description of this subring in Section 2.3.

\subsubsection{The Euler characteristic}

If $M$ is a homogeneous space of the form $G / K$, with $T \subseteq K$, then the odd cohomology of $M$ vanishes and the Euler characteristic of $M$ is equal to

$$
\chi(M)=\sum_{i} \operatorname{dim} H^{2 i}(M)
$$

in particular, the Euler characteristic is non-zero. It is easy to see that the converse is true as well.

Proposition 2.1. If $M=G / K$ and the rank of $K$ is strictly less than the rank of $G$, then the Euler characteristic of $G / K$ is zero.

Proof: Let $h$ be an element of $T$ with the property that

$$
\left\{h^{N} ;-\infty<N<\infty\right\}
$$

is dense in $T$. Suppose that the action of $h$ on $G / K$ fixes a coset $g_{0} K$. Then $g_{0}^{-1} h g_{0} \in K$, i.e. $h$ is conjugate to an element of $K$ and hence conjugate to an element $h_{1}$ of the Cartan subgroup $T_{1}$ of $K$. However, if the iterates of $h$ are dense in $T$, so must be the iterates of $h_{1}$ and hence $T_{1}=T$. Suppose now that $h=\exp \xi, \xi \in \mathfrak{t}$. If $h$ has no fixed points, then 
the vector field $\xi_{M}$ can have no zeroes and hence the Euler characteristic of $M$ has to be zero.

\subsubsection{The fixed points}

We prove in this section that the action of $T$ on $M$ is a GKM action; i.e. that the set of zero dimensional orbits in the orbit space $M / T$ is zero dimensional, and the set of one dimensional orbits is one dimensional. It is easy to see that these properties are equivalent to

(1) $M^{T}$ is finite;

(2) For every codimension one subgroup $H$ of $T, \operatorname{dim} M^{H} \leq 2$.

We will show that if $M$ is of the form $G / K$, with $T \subseteq K$, then it has the two properties above, and we will also show that it has the following third property:

(3) For every subtorus $H$ of $T$ and every connected component $X$ of $M^{H}, X^{T} \neq \emptyset$.

It is well known that these properties hold for the homogeneous space $\mathcal{O}=G / T$. The first two properties can be checked directly (see [9]), and the third property holds because $\mathcal{O}$ is a compact symplectic manifold and the action of $T$ is Hamiltonian. Therefore, to prove that $M$ satisfies Properties 1-3, it suffices to prove the following theorem.

Theorem 2.2. For every subtorus $H$ of $T$, the map

$$
\mathcal{O}=G / T \rightarrow G / K=M
$$

sends $\mathcal{O}^{H}$ onto $M^{H}$.

Proof: Let $p_{0}$ be the identity coset in $M$ and $q_{0}$ the identity coset in $\mathcal{O}$. Let $h$ be an element of $H$ with the property that

$$
\left\{h^{N} ;-\infty<N<\infty\right\}
$$

is dense in $H$. If $p=g p_{0} \in M^{H}$, then $g^{-1} h g \in K$; so $g^{-1} h g=a t a^{-1}$, with $a \in K$ and $t \in T$. Thus $h g a=g a t$ and hence $h q=q$, where $q=g a q_{0}$. But under the map (2.4), $q_{0}$ is sent to $p_{0}$, so $q$ is sent to $g a p_{0}=g p_{0}=p$.

In particular, Theorem 2.2 tells us that the map $\mathcal{O}^{T} \rightarrow M^{T}$ is surjective. However,

$$
\mathcal{O}^{T}=N_{G}(T) / T=W_{G}
$$

so $M^{T}$ is the image of $W_{G}=N_{G}(T) / T$ in $G / K$. But $N_{G}(T) \cap K=N_{K}(T)$, the normalizer of $T$ in $K$, so

$$
\left(N_{G}(T) \cap K\right) / T=W_{K},
$$

and hence we proved: 
Proposition 2.2. There is a bijection

$$
M^{T} \simeq W_{G} / W_{K}
$$

in particular, $W_{G}=N_{G}(T) / T$ acts transitively on $M^{T}$.

\subsubsection{Points stabilized by codimension one subgroups}

Next we compute the connected components of the sets $M^{H}$, where $H$ is a codimension one subgroup of $T$. Let $X$ be one of these components. By Theorem 2.2, $X$ is the image in $M$ of a connected component of $\mathcal{O}^{H}$, and each connected component of $\mathcal{O}^{H}$ is a compact Hamiltonian $T$-space. Therefore its T-fixed point set is non-empty and hence $X^{T} \neq \emptyset$. Moreover, since $M$ is simply connected, it is orientable, and hence every connected component of $M^{H}$ is orientable. So, if $X$ is not an isolated point of $M^{H}$, then it has to be either a circle, a 2-torus, or a 2-sphere, and the first two possibilities are ruled out by the condition $X^{T} \neq \emptyset$. We conclude:

Theorem 2.3. Let $H$ be a codimension one subgroup of $T$ and let $X$ be a connected component of $M^{H}$. Then $X$ is either a point or a 2-sphere.

Remark. By the Korn-Lichtenstein theorem, every faithful action of $S^{1}$ on the 2 -sphere is diffeomorphic to the standard action of "rotation about the $z$-axis." Therefore the action of the circle $S^{1}=T / H$ on the 2-sphere $X$ in the theorem above has to be diffeomorphic to the standard action. In particular, \# $X^{T}=2$.

We now explicitly determine what these 2-spheres are. By Proposition 2.2, each of these 2-spheres is the conjugate by an element of $N_{G}(T)$ of a 2-sphere containing the identity coset $p_{0} \in M=G / K$; so we begin by determining the 2 -spheres containing $p_{0}$.

\subsubsection{The space $\mathfrak{g} / \mathfrak{k}$}

The tangent space $T_{p_{0}} M$ can be identified with $\mathfrak{g} / \mathfrak{k}$, and the isotropy representation of $T$ on this space decomposes into a direct sum of two-dimensional $T$-invariant subspaces

$$
T_{p_{0}} M=\oplus V_{[\alpha]},
$$

labelled by the roots modulo \pm 1 ,

$$
\alpha \in \Delta_{G, K} / \pm 1
$$

One can also regard this as a labelling by the positive roots in $\Delta_{G, K}$; however, since this set of positive roots is not fixed by the natural action of $W_{K}$ on $\Delta_{G, K}$, this is not an intrinsic labelling. (This fact is of importance in Section 3, when we discuss the existence of $G$-invariant almost complex structures on $M$.) Now let $H$ be a codimension one subgroup of $T$, let $\mathfrak{h} \subset \mathfrak{t}$ be the Lie algebra of $H$, and let $M^{H}$ be the set of $H$-fixed points. Then

$$
T_{p_{0}} M^{H}=\left(T_{p_{0}} M\right)^{H} .
$$


Hence, if $X$ is the connected component of $M^{H}$ containing $p_{0}$, and if $X$ is not an isolated point, then $\left(T_{p_{0}} M\right)^{H}$ has to be one of the $V_{[\alpha]}$ 's in the sum (2.5). Hence the adjoint action of $H$ on $\mathfrak{g} / \mathfrak{k}$ has to leave $V_{[\alpha]}$ pointwise fixed. However, an element $g=\exp t$ of $T$ acts on $V_{[\alpha]}$ by the rotation

$$
\chi_{\alpha}(g)=\left(\begin{array}{cc}
\cos \alpha(t) & -\sin \alpha(t) \\
\sin \alpha(t) & \cos \alpha(t)
\end{array}\right)
$$

so the stabilizer group of $V_{[\alpha]}$ is the group

$$
H_{\alpha}=\left\{g \in T ; \chi_{\alpha}(g)=1\right\}
$$

Let $C\left(H_{\alpha}\right)$ be the centralizer of $H_{\alpha}$ in $G$ and let $G_{\alpha}$ be the semisimple component of $C\left(H_{\alpha}\right)$. Then $G_{\alpha}$ is either $S U(2)$ or $S O(3)$, and since $G_{\alpha}$ is contained in $C\left(H_{\alpha}\right), G_{\alpha} p_{0}$ is fixed pointwise by the action of $H$. Moreover, since $G_{\alpha} \nsubseteq \nsubseteq K$, the orbit $G_{\alpha} p_{0}$ can't just consist of the point $p_{0}$ itself; hence

$$
G_{\alpha} p_{0}=X
$$

The Weyl group of $G_{\alpha}$ is contained in the Weyl group of $G$ and consists of two elements: the identity and a reflection, $\sigma=\sigma_{\alpha}$, which leaves fixed the hyperplane $\operatorname{ker} \alpha \subset \mathfrak{t}$, and maps $\alpha$ to $-\alpha$. Therefore, since $\alpha \notin \Delta_{K}, \sigma_{\alpha} p_{0} \neq p_{0}$, and hence $p_{0}$ and $\sigma_{\alpha} p_{0}$ are the two $T$-fixed points on the 2-sphere (2.9).

Now let $p=w p_{0}$ be another fixed point of $T$, with $[w] \in W_{G} / W_{K}$. Let $a$ be a representative for $w$ in $N_{G}(T)$ and let $L_{a}: G \rightarrow G$ be the left action of $a$ on $G$. If $X$ is the 2-sphere (2.9), then the 2-sphere $L_{a}(X)$ intersects $M^{T}$ in the two fixed points $w p_{0}$ and $w \sigma_{\alpha} p_{0}$, and its stabilizer group in $T$ is the group

$$
a H_{\alpha} a^{-1}=w H_{\alpha} w^{-1}=H_{w \alpha},
$$

where $H_{\alpha}$ is the group (2.8).

\subsubsection{The GKM graph of $M$}

This concludes our classification of the set of 2-spheres in $M$ which are stabilized by codimension one subgroups of $T$. Now note that if $X$ is such a two-sphere and $H$ is the subgroup of $T$ stabilizing it, then the orbit space $X / T$ consists of two $T$-fixed points and a connected one dimensional set of orbits having the orbitype of $T / H$. Thus these $X$ 's are in one-toone correspondence with the edges of the GKM graph of $M$. Denoting this graph by $\Gamma$ we summarize the graph-theoretical content of what we've proved so far:

Theorem 2.4. The GKM data associated to the action of $T$ on the homogeneous space $M=G / K$ is the following.

(1) The vertices of $\Gamma$ are in one-to-one correspondence with the elements of $W_{G} / W_{K}$;

(2) Two vertices $[w]$ and $\left[w^{\prime}\right]$ are on a common edge of $\Gamma$ if and only if $\left[w^{\prime}\right]=\left[w \sigma_{\alpha}\right]$ for some $\alpha \in \Delta_{G, K}$; 
(3) The edges of $\Gamma$ containing the vertex $[w]$ are in one-to-one correspondence with the roots, modulo \pm 1 , in the set $\Delta_{G, K}$;

(4) If $\alpha$ is such a root, then the stabilizer group (1.1) labelling the edge corresponding to this root is the group (2.10).

In particular, the labelling (1.1) of the graph $\Gamma$ can be viewed as a labelling by elements $[\alpha]$ of $\Delta_{G} / \pm 1$. We call this labelling a pre-axial function. These graphs may have vertices joined by several distinct edges, as in Figures 1 and 2 (see section 5).

\subsubsection{The connection on $\Gamma$}

One last structural component of the graph $\Gamma$ remains to be described: Given any graph, $\Gamma$, and vertex, $p$, of $\Gamma$, let $E_{p}$ be the set of oriented edges of $\Gamma$ with initial vertex $p$. A connection on $\Gamma$ is a function which assigns to each oriented edge, $e$, a bijective map

$$
\theta_{e}: E_{p} \rightarrow E_{q}
$$

where $p$ is the initial vertex of $e$ and $q$ is the terminal vertex. The graph $\Gamma$ described in Theorem 2.4 has a natural such connection. Namely, let $e$ be an oriented edge of $\Gamma$ joining $[w]$ to $\left[w \sigma_{\alpha}\right]$ and labeled by $[w \alpha]$. If $e^{\prime} \in E_{[w]}$ is an oriented edge joining [w] to $\left[w \sigma_{\beta}\right]$ and labeled by $[w \beta]$, then let $\theta_{e}\left(e^{\prime}\right)=e^{\prime \prime}$, where $e^{\prime \prime}$ is an edge joining $\left[w \sigma_{\alpha}\right]$ to $\left[w \sigma_{\alpha} \sigma_{\beta}\right]$ and labeled by $\left[w \sigma_{\alpha} \beta\right]$. This connection is compatible with the pre-axial function (1.1) in the sense that, for every vertex $p$, and every pair of oriented edges, $e, e^{\prime} \in E_{p}$, the roots labelling $e, e^{\prime}$, and $e^{\prime \prime}=\theta_{e}\left(e^{\prime}\right)$ are coplanar in $\mathrm{t}^{*}$.

\subsubsection{Simplicity}

A graph is said to be simple if every pair of vertices is joined by at most one edge. Most of the graphs above don't have this property. There is however an important class of subgroups, $K$, for which the graph associated with $G / K$ does have this property.

Theorem 2.5. If $K$ is the stabilizer group of an element of $t$, then the graph $\Gamma$ is simple.

Proof: A root $\alpha \in \Delta_{G}$ is in $\Delta_{K}$ if and only if the restriction of $\alpha$ to the subspace $\mathfrak{t}^{W_{K}}$ of $\mathfrak{t}$ is zero. Let $\alpha, \beta \in \Delta_{G, K}$ such that $\alpha \neq \pm \beta$, and let $\sigma_{\alpha}, \sigma_{\beta}$ be the reflections of $\mathfrak{t}$ defined by $\alpha$ and $\beta$. Then $\sigma_{\alpha} \neq \sigma_{\beta}$ and the subspace of $\mathfrak{t}$ fixed by $\sigma_{\alpha} \sigma_{\beta}$ is the codimension 2 subspace on which both $\alpha$ and $\beta$ vanish. If $\sigma_{\alpha} \sigma_{\beta} \in W_{K}$, then this subspace contains $\mathfrak{t}^{W_{K}}$, so $\alpha$ and $\beta$ are both vanishing on $\mathfrak{t}^{W_{K}}$, contradicting our assumption that $\alpha, \beta \notin \Delta_{K}$.

Another way to prove Theorem 2.5 is to observe that $M=G / K$ is a coadjoint orbit of the group $G$. In particular, it is a Hamiltonian $T$-space and $\Gamma$ is the one-skeleton of its moment polytope.

\subsection{The GKM definition of the cohomology ring}

We recall how the data encoded in the GKM graph determines the equivariant cohomology ring $H_{T}^{*}(M)$. The inclusion $i: M^{T} \rightarrow M$ induces a map in cohomology

$$
i^{*}: H_{T}^{*}(M) \rightarrow H_{T}^{*}\left(M^{T}\right)=\operatorname{Maps}\left(M^{T}, \mathbb{S}\left(\mathfrak{t}^{*}\right)\right)=\operatorname{Maps}\left(W_{G} / W_{K}, \mathbb{S}\left(\mathfrak{t}^{*}\right)\right),
$$


and the fact that $M$ is equivariantly formal implies that $i^{*}$ is injective. Let $H_{T}^{*}(\Gamma)$ be the set of maps

$$
f: W_{G} / W_{K} \rightarrow \mathbb{S}\left(\mathfrak{t}^{*}\right)
$$

that satisfy the compatibility condition:

$$
f\left(\left[w \sigma_{\alpha}\right]\right)-f([w]) \in(w \alpha) \mathbb{S}\left(\mathfrak{t}^{*}\right)
$$

for every edge $\left([w],\left[w \sigma_{\alpha}\right]\right)$ of $\Gamma$.

The Goresky, Kottwitz, and MacPherson theorem [6] asserts that

$$
H_{T}^{*}(M) \simeq i^{*}\left(H_{T}^{*}(M)\right)=H_{T}^{*}(\Gamma)
$$

In the next section we construct a direct isomorphism between this ring $H_{T}^{*}(M)$ and the Borel ring given in (2.1).

2.4. Equivalence between the Borel picture and the GKM picture

From the inclusion, $i$, of $M^{T}$ into $M$, one gets a restriction map

$$
i^{*}: H_{T}^{*}(M) \rightarrow H_{T}^{*}\left(M^{T}\right)
$$

and, since $M$ is equivariantly formal, $i^{*}$ maps $H_{T}^{*}(M)$ bijectively onto the subring $H_{T}^{*}(\Gamma)$ of $H_{T}^{*}\left(M^{T}\right)$. However, as we pointed out in Section 2.1,

$$
H_{T}^{*}(M) \simeq \mathbb{S}\left(\mathfrak{t}^{*}\right)^{W_{K}} \otimes_{\mathbb{S}\left(\mathfrak{t}^{*}\right)^{W_{G}}} \mathbb{S}\left(\mathfrak{t}^{*}\right)
$$

so, by combining (2.13) and (2.1), we get an isomorphism

$$
\mathcal{K}: \mathbb{S}\left(\mathfrak{t}^{*}\right)^{W_{K}} \otimes_{\mathbb{S}\left(\mathfrak{t}^{*}\right)^{W_{G}}} \mathbb{S}\left(\mathfrak{t}^{*}\right) \rightarrow H_{T}^{*}(\Gamma)
$$

The purpose of this section is to give an explicit formula for this map. Note that since $M^{T}$ is a finite set,

$$
H_{T}^{*}\left(M^{T}\right)=\bigoplus_{p \in M^{T}} H_{T}^{*}(p)=\bigoplus_{p \in M^{T}} \mathbb{S}\left(\mathfrak{t}^{*}\right)=\operatorname{Maps}\left(M^{T}, \mathbb{S}\left(\mathfrak{t}^{*}\right)\right)
$$

Theorem 2.6. On decomposable elements, $f_{1} \otimes f_{2}$, of the product (2.1),

$$
\mathcal{K}\left(f_{1} \otimes f_{2}\right)=g \in \operatorname{Maps}\left(M^{T}, \mathbb{S}\left(\mathfrak{t}^{*}\right)\right),
$$

where, for $w \in W_{G}$ and $p=w p_{0} \in M^{T}$,

$$
g\left(w p_{0}\right)=\left(w f_{1}\right) f_{2} .
$$


Proof: We first show that (2.15) and (2.16) do define a ring homomorphism of the ring (2.1) into $H_{T}^{*}(\Gamma)$. To show that (2.16) doesn't depend on the representative $w$ chosen, we note that if $w p_{0}=w^{\prime} p_{0}$, then $\sigma=w\left(w^{\prime}\right)^{-1} \in W_{K}$. Thus

$$
g\left(w^{\prime} p_{0}\right)=\left(w^{\prime} f_{1}\right) f_{2}=\left(w \sigma f_{1}\right) f_{2}=\left(w f_{1}\right) f_{2}=g\left(w p_{0}\right)
$$

since $f_{1} \in \mathbb{S}\left(\mathfrak{t}^{*}\right)^{W_{K}}$. Next, we note that if $f \in \mathbb{S}\left(\mathfrak{t}^{*}\right)^{W_{G}}$, then

$$
\mathcal{K}\left(f_{1} f \otimes f_{2}\right)=\mathcal{K}\left(f_{1} \otimes f f_{2}\right),
$$

since

$$
w\left(f_{1} f\right) f_{2}=\left(w f_{1}\right)(w f) f_{2}=\left(w f_{1}\right) f f_{2} .
$$

Thus, by the universality property of tensor products, $\mathcal{K}$ does extend to a mapping of the ring (2.1) into the ring $\operatorname{Maps}\left(M^{T}, \mathbb{S}\left(\mathrm{t}^{*}\right)\right)$.

Next, let $\alpha$ be a root and let $\sigma \in W_{G}$ be the reflection that interchanges $\alpha$ and $-\alpha$ and that is the identity on the hyperplane

$$
\mathfrak{h}=\{\xi \in \mathfrak{t} ; \alpha(\xi)=0\}
$$

Suppose that $p$ and $p^{\prime}$ are two adjacent vertices of $\Gamma$ with $p^{\prime}=\sigma p$. To show that $g=\mathcal{K}\left(f_{1} \otimes f_{2}\right)$ is in $H_{T}^{*}(\Gamma)$, we must show that the quotient

$$
\frac{g\left(p^{\prime}\right)-g(p)}{\alpha}
$$

is in $\mathbb{S}\left(\mathfrak{t}^{*}\right)$. However, if $p=w p_{0}$, then

$$
g\left(p^{\prime}\right)-g(p)=\left(\sigma w f_{1}-w f_{1}\right) f_{2},
$$

and since $\sigma$ is the identity on $\mathfrak{h}$, the restriction of the polynomial $w \sigma f_{1}$ to $\mathfrak{h}$ is equal to the restriction of the polynomial $w f_{1}$ to $\mathfrak{h}$; hence

$$
\frac{g\left(p^{\prime}\right)-g(p)}{\alpha} \in \mathbb{S}\left(\mathfrak{t}^{*}\right)
$$

Next we will show that the domain and range of the map (2.14) are equipped with intrinsic $W_{G}$-actions and that this map is $W_{G}$-equivariant. We first observe that if $M$ is any $G$-manifold, then the action of $N(T)$ on $M$ induces an action of $N(T)$ on $H_{T}^{*}(M)$. If $a \in N(T)$ is an element of the normalizer of $T$ in $G$, then one can define maps $\tau_{a}: M \rightarrow M$ (the action of $a$ on $M$ ) and $\phi_{a}: T \rightarrow T$ (conjugation by $a$ ). Then $\tau_{a}$ is $\phi_{a}$-equivariant, therefore it induces a homomorphism $\psi_{a}: H_{T}^{*}(M) \rightarrow H_{T}^{*}(M)$. The action of $N(T)$ is trivial when restricted to $T$, hence it descends to an action of $W_{G}=N(T) / T$ on $H_{T}^{*}(M)$. We claim that

$$
H_{G}^{*}(M)=H_{T}^{*}(M)^{W_{G}}
$$

(See for instance [8], Theorem 6.8.2). Thus the $G$-equivariant cohomology of $M$ is determined by this action of $W_{G}$ on $H_{T}^{*}(M)$. Conversely, as we pointed out in Section 2.1, there is 
an isomorphism

$$
H_{T}^{*}(M) \simeq H_{G}^{*}(M) \otimes_{\mathbb{S}\left(\mathfrak{t}^{*}\right)^{w_{G}}} \mathbb{S}\left(\mathfrak{t}^{*}\right)
$$

coming from the pullback maps $H_{T}^{*}(p t) \rightarrow H_{T}^{*}(M)$ and $H_{G}^{*}(M) \rightarrow H_{T}^{*}(M)$. The $W_{G}$-action on the right hand side corresponding to the above $W_{G}$-action on $H_{T}^{*}(M)$ is the tensor product of the trivial $W_{G}$-action on the first factor and the intrinsic action of $W_{G}$ on $\mathbb{S}\left(t^{*}\right)$. As corroboration of this fact we note that the $W_{G}$-invariant subring of this tensor product is

$$
H_{G}^{*}(M) \otimes_{\mathbb{S}\left(\mathbf{t}^{*}\right)^{W_{G}}} \mathbb{S}\left(\mathfrak{t}^{*}\right)^{W_{G}}
$$

or $H_{G}^{*}(M)$, as in (2.17). We also observe that since $T$ acts trivially on $M^{T}$ the group, $W_{G}$, acts on $M^{T}$ itself and hence

$$
H_{T}^{*}\left(M^{T}\right)=H^{*}\left(M^{T}\right) \otimes \mathbb{S}\left(\mathfrak{t}^{*}\right)
$$

is a $W_{G}$-module, the action of $W_{G}$ on the right being the tensor product of the induced action of $W_{G}$ on the first factor and the intrinsic action of $W_{G}$ on $\mathbb{S}\left(\mathfrak{t}^{*}\right)$. Finally, we note that if $H_{T}^{*}(M)$ and $H_{T}^{*}\left(M^{T}\right)$ are equipped with these $W_{G}$ actions, then the map (2.13) is a $W_{G}$-module morphism.

Let's apply these remarks to the case at hand, $M=G / K$. As we just observed, if we make the identifications

$$
H_{G}^{*}(M)=\mathbb{S}\left(\mathfrak{t}^{*}\right)^{W_{K}}
$$

and

$$
H_{T}^{*}(M)=\mathbb{S}\left(\mathfrak{t}^{*}\right)^{W_{K}} \otimes_{\mathbb{S}\left(\mathfrak{t}^{*}\right)^{W_{G}}} \mathbb{S}\left(\mathfrak{t}^{*}\right)
$$

the action of $W_{G}$ on $H_{T}^{*}(M)$ is the action defined by

$$
w\left(f_{1} \otimes f_{2}\right)=f_{1} \otimes w f_{2}
$$

and, if we make the identifications

$$
M^{T}=W_{G} / W_{K}
$$

and

$$
H_{T}^{*}\left(M^{T}\right)=\operatorname{Maps}\left(M^{T}, \mathbb{S}\left(\mathrm{t}^{*}\right)\right)
$$

the action of $W_{G}$ on $H_{T}^{*}\left(M^{T}\right)$ is the action

$$
f^{w}(p)=w f\left(w^{-1} p\right)
$$

and these two actions are intertwined by the map (2.14). To show that the map, $\mathcal{K}$, defined by (2.15) and (2.16) coincides with (2.14) we will first show that it too intertwines these two 은 Springer 
actions. In other words we will show that if

$$
g=\mathcal{K}\left(f_{1} \otimes f_{2}\right) \quad \text { and } g^{w}=\mathcal{K}\left(f_{1} \otimes w f_{2}\right),
$$

then for all points $p=\sigma p_{0}$,

$$
g^{w}(p)=(w g)(p)
$$

However,

$$
g^{w}(p)=\left(\sigma f_{1}\right)\left(w f_{2}\right)=w\left(\left(w^{-1} \sigma f_{1}\right) f_{2}\right)=w g\left(w^{-1} p\right)=(w g)(p) .
$$

Let us now prove that the map $\mathcal{K}$ coincides with the map (2.14). We first note that $\mathcal{K}$ is a morphism of $\mathbb{S}\left(\mathfrak{t}^{*}\right)$-modules. For $f \in \mathbb{S}\left(\mathfrak{t}^{*}\right)$,

$$
\mathcal{K}\left(f_{1} \otimes f_{2} f\right)=\mathcal{K}\left(f_{1} \otimes f_{2}\right) f
$$

Thus, it suffices to verify that $\mathcal{K}$ agrees with the map (2.14) on elements of the form $f_{1} \otimes 1$. That is, in view of the identification (2.14), it suffices to show that $\mathcal{K}$, restricted to $\mathbb{S}\left(\mathrm{t}^{*}\right)^{W_{K}} \otimes$ 1 , agrees with the map (2.14), restricted to $H_{T}^{*}(M)^{W_{G}}$. However, if $f \in H_{T}^{*}(M)^{W_{G}}$, then $i^{*} f \in H_{T}^{*}\left(M^{T}\right)^{W_{G}}$, so it suffices to show that $i^{*} f$ and $\mathcal{K}(f \otimes 1)$ coincide at $p_{0}$, the identity coset of $M=G / K$. This is equivalent to showing that in the diagram below

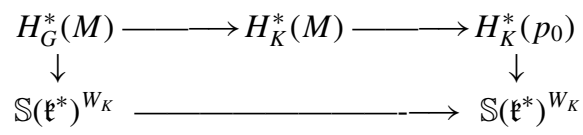

the bottom arrow is the identity map. However, the bottom arrow is clearly the identity on $\mathbb{S}^{0}\left(\mathfrak{k}^{*}\right)^{K}=\mathbb{C}$ and the two maps on the top line are $\mathbb{S}\left(\mathfrak{k}^{*}\right)^{K}$-module morphisms.

\section{Almost complex structures and axial functions}

\subsection{Axial functions}

A $G$-invariant almost complex structure on $M=G / K$ is determined by an almost complex structure on the tangent space $T_{p_{0}} M$,

$$
J_{p_{0}}: T_{p_{0}} M \simeq \mathfrak{g} / \mathfrak{k} \rightarrow \mathfrak{g} / \mathfrak{k} .
$$

For an arbitrary point $g p_{0} \in M$, the almost complex structure on

$$
T_{g p_{0}} M=\left(d L_{g}\right)_{p_{0}}\left(T_{p_{0}} M\right)
$$

is given by

$$
J_{g p_{0}}\left(\left(d L_{g}\right)_{p_{0}}(X)\right)=\left(d L_{g}\right)_{p_{0}}\left(J_{p_{0}}(X)\right)
$$


for all $X \in \mathfrak{g} / \mathfrak{k}$. This definition is independent on the representative $g$ chosen if and only if $J_{p_{0}}$ is $K$-invariant. Therefore $G$-invariant almost complex structures on $G / K$ are in one to one correspondence to $K$-invariant almost complex structures on $\mathfrak{g} / \mathfrak{k}$.

If $M=G / K$ has a $G$-invariant almost complex structure, then the isotropy representations of $T$ on $T_{p_{0}} M$ is a complex representation, and therefore its weights are well-defined (not just well-defined up to sign). Let

$$
T_{p_{0}} M=\mathfrak{g} / \mathfrak{k}=\bigoplus_{[\beta]} V_{[\beta]}
$$

be the root space decomposition of $\mathfrak{g} / \mathfrak{k}$. Then $V_{[\beta]}$ is a one-dimensional complex representation of $T$; let $\widetilde{\beta} \in\{ \pm \beta\}$ be the weight of this complex representation:

$$
\exp t \cdot X_{\widetilde{\beta}}=e^{i \widetilde{\beta}(t)} X_{\widetilde{\beta}}, \quad \text { for all } t \in \mathfrak{t} .
$$

Thus, the map

$$
s: \Delta_{G, K} / \pm 1 \rightarrow \Delta_{G, K}, s([\beta])=\widetilde{\beta}
$$

is a $W_{K}$-equivariant right inverse of the projection $\Delta_{G, K} \rightarrow \Delta_{G, K} /\{ \pm 1\}$. Let $\Delta_{0} \subset \Delta_{G, K}$ be the image of $s$.

The existence of a map (3.1) is equivalent to the condition

$$
w \alpha \neq-\alpha \quad \text {, for all } w \in W_{K}, \alpha \in \Delta_{G, K}=\Delta_{G}-\Delta_{K},
$$

hence (3.2) is a necessary condition for the existence of a $G$-invariant almost complex structure on $M$. We will see in the next section that this condition is also sufficient.

We can now define a labelling of the oriented edges, $E_{\Gamma}$, of the GKM graph $\Gamma$, as follows. Let $[w] \in W_{G} / W_{K}$ be a vertex of the graph and let $e=\left([w],\left[w \sigma_{\beta}\right]\right)$ be an oriented edge of the graph, with $\beta \in \Delta_{0}$. This edge corresponds to the subspace $V_{[w \beta]}$ (see (2.10)) in the decomposition

$$
T_{[w]} M=\bigoplus_{\beta \in \Delta_{0}} V_{[w \beta]},
$$

and the $G$-invariance of the almost complex structure implies that $T$ acts on $V_{[w \beta]}$ with weight $w \beta$. We define $\alpha: E_{\Gamma} \rightarrow \mathfrak{t}^{*}$ by

$$
\alpha\left([w],\left[w \sigma_{\beta}\right]\right)=w \beta \text {, for all } \beta \in \Delta_{0}, w \in W_{G} .
$$

Theorem 3.1. The map $\alpha: E_{\Gamma} \rightarrow t^{*}$ has the following properties:

(1) If $e_{1}$ and $e_{2}$ are two oriented edges with the same initial vertex, then $\alpha\left(e_{1}\right)$ and $\alpha\left(e_{2}\right)$ are linearly independent;

(2) If $e$ is an oriented edge and $\bar{e}$ is the same edge, with the opposite orientation, then $\alpha(\bar{e})=-\alpha(e)$

(3) If e and $e^{\prime}$ are oriented edge with the same initial vertex, and if $e^{\prime \prime}=\theta_{e}\left(e^{\prime}\right)$, then $\alpha\left(e^{\prime \prime}\right)$ $-\alpha\left(e^{\prime}\right)$ is a multiple of $\alpha(e)$. 
Proof: The first assertion is a consequence of the fact that the only multiples of a root $\alpha$ that are roots are $\pm \alpha$.

If $e$ is the oriented edge that joins $[w]$ to $\left[w \sigma_{\beta}\right]$ and that is labelled by $w \beta \in w \Delta_{0}$, then

$$
\alpha(\bar{e})=\left(w \sigma_{\beta}\right)(\beta)=-w \beta=-\alpha(\bar{e}) .
$$

Finally, if $e$ joins $[w]$ to $\left[w \sigma_{\beta}\right]$ and if $e^{\prime}$ joins $[w]$ to $\left[w \sigma_{\gamma}\right]$ (with $\beta, \gamma \in \Delta_{0}$ ), then $e^{\prime \prime}$ joins $\left[w \sigma_{\beta}\right]$ to $\left[w \sigma_{\beta} \sigma_{\gamma}\right]$, and

$$
\alpha\left(e^{\prime \prime}\right)-\alpha(e)=w \sigma_{\beta} \gamma-w \gamma=w\left(\sigma_{\beta} \gamma-\gamma\right)=-\langle\gamma, \beta\rangle w \beta=-\langle\gamma, \beta\rangle \alpha(e) .
$$

Equivalently, Theorem 3.1 says that $\alpha: E_{\Gamma} \rightarrow \mathfrak{t}^{*}$ is an axial function compatible with the connection $\theta$, in the sense of [9].

\subsection{Invariant almost complex structures}

As we have seen in Section 3.1, (3.2) is a necessary condition for the existence of a $G$-invariant almost complex structure on $M=G / K$; in this section we show that it also a sufficient condition.

\section{Theorem 3.2. If the condition}

$$
w \alpha \neq-\alpha, \quad \text { for all } w \in W_{K}, \alpha \in \Delta_{G, K}=\Delta_{G}-\Delta_{K},
$$

is satisfied, then $M$ admits a G-invariant almost complex structure.

Proof: Consider the complex representation of $K$ on $(\mathfrak{g} / \mathfrak{k})_{\mathbb{C}}=\mathfrak{g}_{\mathbb{C}} / \mathfrak{k}_{\mathbb{C}}$ and let

$$
(\mathfrak{g} / \mathfrak{k})_{\mathbb{C}}=\bigoplus_{j} V_{j}
$$

be the decomposition into irreducible representations; $(\mathfrak{g} / \mathfrak{k})_{\mathbb{C}}$ is self dual, hence

$$
\bigoplus_{j} V_{j}=(\mathfrak{g} / \mathfrak{k})_{\mathbb{C}}=(\mathfrak{g} / \mathfrak{k})_{\mathbb{C}}^{*}=\bigoplus_{j} V_{j}^{*}=\bigoplus_{j} \overline{V_{j}}
$$

Therefore $\overline{V_{j}}=V_{l}$ for some $l$. If $\alpha$ is a highest weight of $V_{j}$, then condition (3.2) implies that $-\alpha$ is not a weight of $V_{j}$; however, $-\alpha$ is a weight of $\overline{V_{j}}$, hence $\overline{V_{j}} \neq V_{j}$. Therefore

$$
(\mathfrak{g} / \mathfrak{k})_{\mathbb{C}}=\bigoplus_{j}\left(V_{j} \oplus \overline{V_{j}}\right)=U \oplus \bar{U}
$$

as complex $K$-representations, and this induces a $K$-invariant almost complex structure

$$
J: \mathfrak{g} / \mathfrak{k} \rightarrow \mathfrak{g} / \mathfrak{k}
$$


as follows: If $x \in \mathfrak{g} / \mathfrak{k}$, then there exists a unique $y \in \mathfrak{g} / \mathfrak{k}$ such that $x+i y \in U$, and we define $J(x)=y$. As we have shown before, this is equivalent to the existence of a $G$-invariant almost complex structure on $M$.

An alternative way of proving Theorem 3.2 is to observe that the condition (3.2) is equivalent to the existence of a $W_{K}$-equivariant section $s: \Delta_{G, K} / \pm 1 \rightarrow \Delta_{G, K}$. Let $s$ be such a section and let $\Delta_{0} \subset \Delta_{G}-\Delta_{K}$ be the image of $s$. Then (see (2.5))

$$
\mathfrak{g} / \mathfrak{k}=\bigoplus_{\alpha \in \Delta_{0}} V_{[\alpha]}
$$

and one can define a $K$-invariant almost complex structure $J$ by requiring that for each $\alpha \in \Delta_{0}, J$ acts on $V_{[\alpha]}$ by

$$
J\left(\begin{array}{c}
X_{\alpha} \\
X_{-\alpha}
\end{array}\right)=\left(\begin{array}{c}
X_{-\alpha} \\
-X_{\alpha}
\end{array}\right)
$$

\section{Morse theory on the GKM graph}

\subsection{Betti numbers}

Henceforth we assume that $M$ admits a $G$-invariant almost complex structure, determined (see (3.4)) by the image $\Delta_{0} \subset \Delta_{G, K}$ of a section $s: \Delta_{G, K} / \pm 1 \rightarrow \Delta_{G, K}$. Let $\Gamma$ be the GKM graph of $M$ and let

$$
\alpha: E_{\Gamma} \rightarrow \mathfrak{t}^{*}
$$

be the axial function (3.3). Then the edges whose initial vertex is the identity coset in $W_{G} / W_{K}$ are labelled by vectors in $\Delta_{0}$.

Let $\xi \in \mathfrak{t}$ be a regular element of $\mathfrak{t}$, i.e.

$$
\beta(\xi) \neq 0, \quad \text { for all } \beta \in \Delta_{G} \subset \mathfrak{t}^{*} .
$$

For a vertex $[w] \in W_{G} / W_{K}$, let $E_{[w]}$ be the set of oriented edges issuing from [ $\left.w\right]$. We define the index of $[w]$ to be

$$
\operatorname{ind}_{[w]}=\#\left\{e \in E_{[w]} ; \alpha(e)(\xi)<0\right\},
$$

and for each $k \geq 0$, let the $k$-th Betti number of $\Gamma$ be defined by

$$
\beta_{k}(\Gamma)=\#\left\{[w] \in W_{G} / W_{K} ; \operatorname{ind}_{[w]}=k\right\}
$$

The index of a vertex obviously depends on $\xi$, but the Betti numbers do not.

Theorem 4.1([9]). The Betti numbers $\beta_{k}(\Gamma)$ are combinatorial invariants of $\Gamma$ (i.e. are independent of $\xi$ ).

은 Springer 
In general these Betti numbers are not equal to the Betti numbers

$$
\beta_{2 k}(M)=\operatorname{dim} H^{2 k}(M)
$$

of $M=G / K$; see Example 5.2. However, we show in the next section that there is a large class of homogeneous spaces for which they are equal.

\subsection{Morse functions}

Let $\xi \in \mathfrak{t}$ be a regular element.

Definition 4.1. A function $f: W_{G} / W_{K} \rightarrow \mathbb{R}$ is called a Morse function compatible with $\xi$ if for every oriented edge $e=\left([w],\left[w^{\prime}\right]\right)$ of the GKM graph, the condition $f\left(\left[w^{\prime}\right]\right)>f([w])$ is satisfied whenever $\alpha(e)(\xi)>0$.

Morse function do not always exist; however, there is a simple necessary and sufficient condition for the existence of a Morse function: Every regular element $\xi \in \mathfrak{t}$ determines an orientation $o_{\xi}$ of the edges of $\Gamma$ : an edge $e \in E_{\Gamma}$ points upward (with respect to $\xi$ ) if $\alpha_{e}(\xi)>0$, and points downward if $\alpha_{e}(\xi)<0$. The associated directed graph $\left(\Gamma, o_{\xi}\right)$ is the graph with all upward-pointing edges.

Proposition 4.1. There exists a Morse function compatible with $\xi$ if and only if the directed graph $\left(\Gamma, o_{\xi}\right)$ has no cycles.

\subsection{Invariant complex structures}

In this section we show that the existence of Morse functions on the GKM graph, which is a combinatorial condition, has geometric implications for the space $M=G / K$.

Theorem 4.2. The GKM graph $(\Gamma, \alpha)$ admits a Morse function compatible with a regular $\xi \in \mathfrak{t}$ if and only if the almost complex structure determined by $\alpha$ is a $K$-invariant complex structure on M. Moreover, if this is the case, then the combinatorial Betti numbers agree with the topological Betti numbers. That is,

$$
b_{k}(\Gamma)=b_{2 k}(M)
$$

Proof: Let $f: W_{G} / W_{K} \rightarrow \mathbb{R}$ be a Morse function compatible with $\xi$, and let [ $\left.w\right]$ be a vertex of the GKM graph where $f$ attains its minimum. If we replace $\xi$ by $w^{-1}(\xi)$ and $f$ by $\left(w^{-1}\right)^{*} f$, then the minimum of this new function is $p_{0}$. Thus, without loss of generality, we may assume that the minimum vertex $[w]$ is the identity coset in $W_{G} / W_{K}$. Then

$$
\Delta_{0}=\left\{\beta \in \Delta_{G, K} ; \beta(\xi)>0\right\} .
$$

Let $\mathfrak{g}_{\mathbb{C}}$ be the complexification of $\mathfrak{g}$ and, for $\beta \in \Delta_{0}$, let $\mathfrak{g}_{\beta}$ be the one-dimensional complex root space. The root space $\mathfrak{g}_{\beta}$ corresponds to invariant vector fields on $M$ which are holomorphic with respect to the almost complex structure defined by $\Delta_{0}$. Since $\left[\mathfrak{g}_{\beta_{1}}, \mathfrak{g}_{\beta_{2}}\right] \subset \mathfrak{g}_{\beta_{1}+\beta_{2}}$ and $\Delta_{0}+\Delta_{0} \subset \Delta_{0}$, it follows that the invariant almost complex structure defined by $\Delta_{0}$ is integrable, hence it is an invariant complex structure on $M$. 
Let

$$
\mathfrak{p}=\mathfrak{k}_{\mathbb{C}} \oplus\left(\bigoplus_{\beta \in \Delta_{0}} \mathfrak{g}_{\beta}\right)
$$

Then $\mathfrak{p}$ is a parabolic subalgebra of $\mathfrak{g}_{\mathbb{C}}$. If $G_{\mathbb{C}}$ is the simply connected Lie group with Lie algebra $\mathfrak{g}_{\mathbb{C}}$ and $P$ is the Lie subgroup of $G_{\mathbb{C}}$ corresponding to $\mathfrak{p}$, then

$$
M=G / K \simeq G_{\mathbb{C}} / P
$$

hence $M$ is a flag variety. Then $M$ is a Hamiltonian $T$-space and the GKM graph of $M$ is the 1 -skeleton of the moment polytope. If $\mu: M \rightarrow t^{*}$ is the moment map, then $\mu^{\xi}: M \rightarrow \mathbb{R}$ is a Morse function on $M$ whose critical points are the fixed points $M^{T}$. The index of $\mu^{\xi}$ at a point $p \in M^{T}$ is twice the index of the vertex of $\Gamma$ corresponding to $p$. Therefore the combinatorial Betti numbers agree with the topological Betti numbers.

On the other hand, if the almost complex structure is integrable then $\mathfrak{p}$ is a parabolic subalgebra of $\mathfrak{g}_{\mathbb{C}}, M=G / K \subset \mathfrak{g}^{*}$ is a coadjoint orbit of $G$, and for a generic direction $\xi \in \mathfrak{t} \subset \mathfrak{g}$, the map $f: W_{G} / W_{K} \rightarrow \mathbb{R}$ given by

$$
f([w])=\langle[w], \xi\rangle
$$

(with $W_{G} / W_{K} \rightarrow G / K \rightarrow \mathfrak{g}^{*}$ ) is a Morse function on the GKM graph compatible with $\xi$.

\section{Examples}

\subsection{Non-existence of almost complex structures}

Let $G$ be a compact Lie group such that $\mathfrak{g}_{\mathbb{C}}$ is the simple Lie algebra of type $B_{2}$. Let $\alpha_{1}, \alpha_{1}+$ $\alpha_{2}$ be the short positive roots and let $\alpha_{2}, \alpha_{2}+2 \alpha_{1}$ be the long positive roots. Let $K$ be the subgroup of $G$ corresponding to the root system consisting of the short roots. Then $\mathfrak{k}_{\mathbb{C}}=D_{2}=A_{1} \times A_{1}$ and $K \simeq S U(2) \times S U(2)$. The quotient $W_{G} / W_{K}$ has two classes: the class of $\sigma_{\alpha_{1}} \in W_{K}$ and the class of $\sigma_{\alpha_{2}} \in W_{G}-W_{K}$.

The GKM graph $\Gamma$ has two vertices, joined by two edges, and the edges are labelled by $\left[\alpha_{2}\right],\left[\alpha_{2}+2 \alpha_{1}\right] \in \Delta_{G, K} / \pm 1$ (see Figure 1). If $w=\sigma_{\alpha_{1}+\alpha_{2}} \sigma_{\alpha_{1}} \in W_{K}$, then $w \alpha_{2}=-\alpha_{2}$ and $\alpha_{2} \in \Delta_{G, K}$, hence one can't define an axial function on $\Gamma$. In this example, $G / K=S^{4}$, which does not admit an almost complex structure [12, paragraph 41.20].
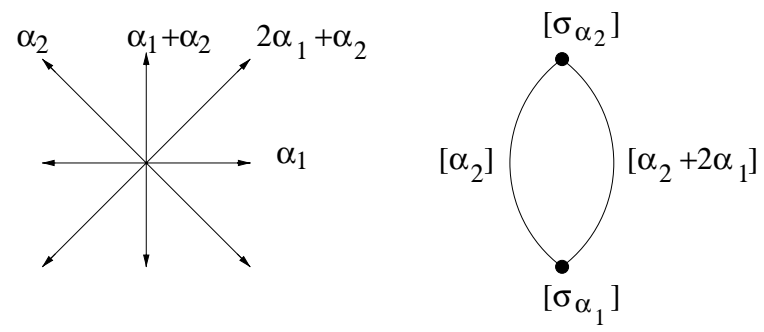

Fig. 1 The root system for $S O(5)$ and the GKM graph for the homogeneous space $S O(5) /(S U(2) \times S U(2))$ 

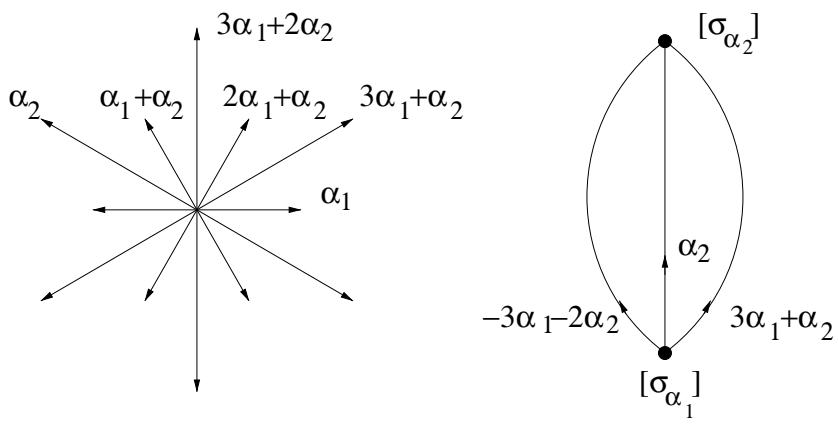

Fig. 2 The root system for $G_{2}$ and the GKM graph for the homogeneous space $G_{2} / G U(3)$

\subsection{Non-existence of Morse functions}

Let $G$ be a compact Lie group such that $\mathfrak{g}_{\mathbb{C}}$ is the simple Lie algebra of type $G_{2}$. Let $\alpha_{1}, \alpha_{1}+\alpha_{2}$, and $2 \alpha_{1}+\alpha_{2}$ be the short positive roots and let $\alpha_{2}, 2 \alpha_{2}+3 \alpha_{1}, \alpha_{2}+3 \alpha_{1}$ be the long positive roots. Let $K$ be the subgroup of $G$ corresponding to the root system consisting of the short roots. Then $\mathfrak{k}_{\mathbb{C}}=A_{2}$ and $K \simeq S U(3)$. The quotient $W_{G} / W_{K}$ has two classes: the class of $\sigma_{\alpha_{1}} \in W_{K}$ and the class of $\sigma_{\alpha_{2}} \in W_{G}-W_{K}$.

The GKM graph $\Gamma$ has two vertices, joined by three edges, and the edges are labelled by $\left[\alpha_{2}\right],\left[2 \alpha_{2}+3 \alpha_{1}\right],\left[\alpha_{2}+3 \alpha_{1}\right] \in \Delta_{G, K} / \pm 1$. There are two $W_{K}$-equivariant sections of the projection $\Delta_{G, K} \rightarrow \Delta_{G, K} / \pm 1$, corresponding to $\left\{\alpha_{2}, \alpha_{2}+3 \alpha_{1},-2 \alpha_{2}-3 \alpha_{1}\right\}$ and $\left\{-\alpha_{2},-\alpha_{2}-3 \alpha_{1}, 2 \alpha_{2}+3 \alpha_{1}\right\}$. If

$$
\Delta_{0}=\left\{\alpha_{2}, \alpha_{2}+3 \alpha_{1},-2 \alpha_{2}-3 \alpha_{1}\right\}
$$

then the axial function is shown in Figure 2 and there is no Morse function on $\Gamma$ : the corresponding almost complex structure is not integrable. In this example, $G / K=S^{6}$, which admits an almost complex structure, but no invariant complex structure.

\subsection{The existence of several almost complex structures}

Let $G=S U(3)$ and $K=T$. Then the homogeneous space $G / K$ is the manifold of complete flags in $\mathbb{C}^{3}$. The root system of $G$ is $A_{2}$, with positive roots $\alpha_{1}, \alpha_{2}$, and $\alpha_{1}+\alpha_{2}$ of equal length. The Weyl group of $G$ is $W_{G}=S_{3}$, the group of permutations of $\{1,2,3\}$, and $W_{K}=1$, hence $W_{G} / W_{K}=W_{G}=S_{3}$.

The GKM graph is the bi-partite graph $K_{3,3}$ : it has 6 vertices and each vertex has 3 edges incident to it, labelled by $\left[\alpha_{1}\right],\left[\alpha_{2}\right]$, and $\left[\alpha_{1}+\alpha_{2}\right]$. There are $2^{3}$ possible $W_{K}$-invariant sections, hence eight $G$-invariant almost complex structures on $G / K$. If

$$
\Delta_{0}=\left\{\alpha_{1}, \alpha_{2}, \alpha_{1}+\alpha_{2}\right\}
$$

then the corresponding almost complex structure is integrable and there is a Morse function on $\Gamma$ compatible with $\xi \in \mathfrak{t}$ such that both $\alpha_{1}(\xi)$, and $\alpha_{2}(\xi)$ are positive. A Morse function is given by $f(w)=\ell(w)$, where $\ell(w)$ is the length of $w$. In this case, $\ell(w)$ is the same as the number of inversions in $w$ (see [10], p. 13). For example, the transposition (321) has length three and has three inversions, corresponding to positions $(1,2),(1,3)$, and $(2,3)$. 


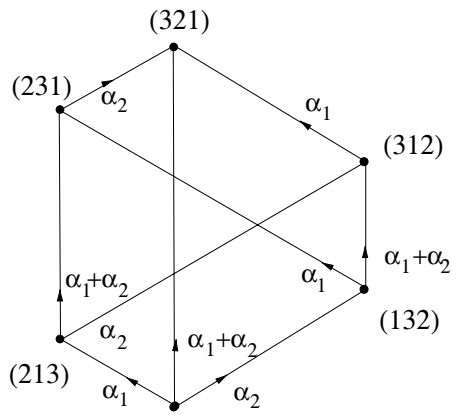

(123)

(a)

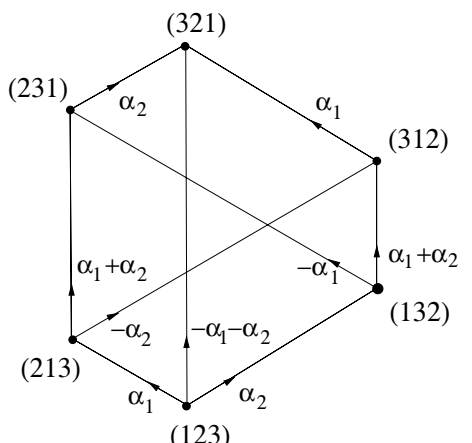

(b)

Fig. 3 GKM graphs corresponding to integrable and non-integrable almost complex structures on $S U(3) / T$

However, if

$$
\Delta_{0}=\left\{\alpha_{1}, \alpha_{2},-\alpha_{1}-\alpha_{2}\right\}
$$

then the corresponding almost complex structure is not integrable and there is no Morse function on $(\Gamma, \alpha)$ : for every vertex $w$ of $\Gamma$, there exist three edges $e_{1}, e_{2}$, and $e_{3}$, going out of $w$, such that

$$
\alpha_{e_{1}}+\alpha_{e_{2}}+\alpha_{e_{3}}=0
$$

hence there is no vertex of $\Gamma$ on which a Morse function compatible with some $\xi \in \mathfrak{t}$ can achieve its minimum. These two examples are shown in Figure 3.

In general, if $G$ is a compact, connected, semisimple Lie group and $T$ is a maximal torus, then the number of $G$-invariant almost complex structures on $G / T$ is $2^{r}$, where $r$ is the number of positive roots. The integrable almost complex structures correspond bijectively to systems of positive roots, hence there are $\# W_{G}$ invariant complex structures.

Acknowledgments We are grateful to David Vogan for helping us formulate and prove the results in Section 3 and to Bert Kostant for pointing out to us that a homogeneous space is a quotient of a compact group by a closed subgroup of the same rank if and only if its Euler characteristic is non-zero, and for making us aware of a number of nice properties of such spaces.

\section{References}

1. A. Borel, "Seminar on transformation groups," Ann. of Math. Stud. 46, Princeton Univ. Press, Princeton, NJ 1960.

2. A. Borel and J. De Siebenthal, "Les sous-groupes fermés de rang maximum des groupes de Lie clos (French)," Comment. Math. Helv. 23 (1949), 200-221.

3. W. Fulton and J. Harris, Representation theory, Springer, New York, 1991.

4. W. Greub, S. Halperin, and R. Vanstone, Connections, curvature, and cohomology, vol. II, Academic Press, 1973.

5. W. Greub, S. Halperin, and R. Vanstone, Connections, curvature, and cohomology, vol. III, Academic Press, 1976. 
6. M. Goresky, R. Kottwitz and R. MacPherson, "Equivariant cohomology, Koszul duality, and the localization theorem," Invent. Math. 131(1) (1998), 25-83.

7. R. Goldin, The cohomology of weight varieties, Ph.D. thesis, MIT 1999.

8. V. Guillemin and S. Sternberg, Supersymetry and equivariant de Rham cohomology, Springer Verlag, Berlin, 1999.

9. V. Guillemin and C. Zara, "One-skeleta, Betti numbers and equivariant cohomology," Duke Math. J. 107(2) (2001), 283-349.

10. J. Humphreys, Reflection groups and Coxeter groups, Cambridge Univ. Press, 1990.

11. T. Holm, "Homogeneous spaces, equivariant cohomology, and graphs," Ph.D. thesis, MIT 2002.

12. N. Steenrod, The topology of fibre bundles, Princeton University Press, 1951.

13. D. Vogan, personal communication. 